\title{
Commentary: Association of autoimmune hepatitis and Evans syndrome in children
}

\author{
Chaowapong Jarasvaraparn', David A. Gremse ${ }^{2 *}$
}

'Department of Pediatrics, University of South Alabama, Mobile, Alabama 36604, USA

${ }^{2}$ Division of Pediatric Gastroenterology, Hepatology and Nutrition, University of South Alabama, Mobile, Alabama 36604, USA

Article Info

\section{Article Notes}

Received: October 30, 2017

Accepted: December 05, 2017

\section{*Correspondence:}

Dr. David A. Gremse, Division of Pediatric Gastroenterology, Hepatology and Nutrition, University of South Alabama, Mobile, Alabama 36604, USA, 1601 Center St, Mobile, AL 36604, Telephone: +1-251-434-3919

Fax: +1-251-434-3980

(c) 2017 Gremse DA. This article is distributed under the terms of the Creative Commons Attribution 4.0 International License.

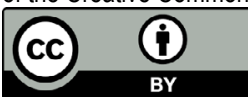

\section{Keywords}

Autoimmune hepatitis type 1

Evans syndrome

Children

\section{ABSTRACT}

Autoimmune hepatitis (AlH) is a chronic liver disease that may be associated with extrahepatic autoimmune disorders. Evans syndrome (ES) is an autoimmune disorder that is characterized by a combination of immune thrombocytopenia and autoimmune hemolytic anemia. Association of autoimmune hepatitis with Evans syndrome is rare, especially in children. We reported a 3-year-old-female with pre-existing Evans syndrome who developed AlH type $1 .{ }^{1}$ This commentary reviews this case along with other reported cases of AlH and ES.

\section{Introduction}

AIH is a chronic necroinflammatory liver disease that is associated with circulating autoantibodies and hypergammaglobulinemia. AIH occurs predominantly in females (female:male ratio $=3: 1$ ) and most patients are diagnosed before the age of 18 years with a peak incidence prior to puberty. AIH type 1 is diagnosed for patients exhibiting seropositivity for smooth muscle and/or anti-nuclear antibodies while AIH type 2 is defined for those with liver kidney microsomal antibodies and/or liver cytosol antigen. AIH type 1 accounts for two-thirds of the cases and presents usually during adolescence, whereas AIH type 2 presents at a younger age especially during infancy ${ }^{2}$. In children, AIH is more likely to present acutely with a more aggressive course than in adults ${ }^{3}$. If left untreated, it may progress rapidly to cirrhosis and end stage liver disease.

ES is a rare autoimmune disease that was first described in 1951, characterized by a combination of immune thrombocytopenia (ITP) and autoimmune hemolytic anemia (AIHA). Both diseases are mediated by autoantibodies, though in some cases it is considered a T-lymphocyte disorder. ${ }^{4}$ As typical of other auto-immune disorders, ES has a chronic and relapsing course and treatment requires prolonged immunosuppressive therapy since it is associated with a higher mortality risk than AIHA alone ${ }^{5}$. Although approximately half of patients with ES have no other associated immune disorders, ES has been reported in association with other conditions such as systemic lupus erythematosus, common variable immune deficiency ${ }^{6}$, or autoimmune lymphoproliferative disorder ${ }^{7,8}$. ES in children is considered a serious condition because the risk of life threatening hemorrhage is greater than in classic ITP ${ }^{4}$. 
AIH has also been reported with other autoimmune disorders such as systemic lupus erythematosus, rheumatoid arthritis, Sjögren's syndrome, chronic thyroiditis, ulcerative colitis, celiac disease, connective tissue diseases, membranoproliferative glomerulonephritis, myasthenia gravis and immune thrombocytopenia. The association of AIH with ES is rare, especially in children. We reported the case of a child with AIH and ES ${ }^{1}$. We speculate that autoimmune diseases, such as AIH and ES, may share genetic susceptibility factors.

\section{Case Report}

A previously healthy two-year-old African American female was diagnosed with ES after a two-month history of epistaxis and easy bruising ${ }^{1}$. Laboratory evaluation was significant for anemia with hemoglobin $8.4 \mathrm{~g} / \mathrm{dl}$, and thrombocytopenia with a platelet count of $61,000 /$ $\mu \mathrm{l}$. Testing for associated viral infection with hepatitis $A$, B, or C, cytomegalovirus, Epstein-Barr virus, parvovirus, or herpes simplex virus were negative. Her antiplatelet antibody, direct Coomb's test and anti-compliment factor 3 antibody were positive and her anti-nuclear antibody (ANA) was negative. She responded to treatment with a single $1 \mathrm{~g} / \mathrm{kg}$ dose of intravenous immunoglobulin (IVIg) followed by oral prednisone at $2 \mathrm{mg} / \mathrm{kg} /$ day.

One year later, she developed $\mathrm{AIH}$ presenting with jaundice, pruritus, hepatomegaly, transaminitis with aspartate aminotransferase (AST) 547 IU/L and alanine transaminase (ALT) $600 \mathrm{IU} / \mathrm{L}$, hypoalbuminemia with albumin $2.6 \mathrm{~g} / \mathrm{dl}$, with normal total serum protein of $7.9 \mathrm{~g} / \mathrm{dL}$. She had cholestatic jaundice with a total and direct bilirubin of $10.2 \mathrm{mg} / \mathrm{dl}$ and $8.8 \mathrm{mg} /$ $\mathrm{dl}$, respectively. The serum alpha-1-antitrypsin and ceruloplasmin concentrations were normal. Autoimmune markers were positive for ANA (1:40), smooth muscle antibody (1:40), and $\mathrm{F}$ actin antibody (39 units) with hypergammaglobulinemia (total serum IgG 1,090 mg/ dL). Her anti-liver-kidney-microsome antibody, anti-HAVIgM, HBsAg, anti-HBc and anti-HCV were negative. Liver biopsy confirmed histologic evidence of AIH with interface hepatitis of a mixed inflammatory infiltrate including lymphoid cells, eosinophils, neutrophils, histiocytic cells and plasma cells in addition to periportal fibrosis with rare portal-portal septa (stage 2 fibrosis) along with canalicular and hepatocytic cholestasis. Immunologic screening tests showed normal immunoglobulin concentrations, normal absolute lymphocyte counts and sub-set populations (including CD3, CD4, CD8, CD56, no double negative $\mathrm{T}$ cells) via flow cytometry without evidence of an autoimmune lymphoproliferative disorder. She responded to treatment with corticosteroids and azathioprine. During her hospitalization for AIH, she had an Evans syndrome exacerbation, with a drop in hemoglobin to $4.9 \mathrm{~g} / \mathrm{dl}$ and elevated reticulocyte count up to $44 \%$ with normal platelet count which was treated with intravenous rituximab 375 $\mathrm{mg} / \mathrm{m} 2$ weekly for four doses followed by IVIg monthly for six months. She responded with an increased hemoglobin to $14 \mathrm{~g} / \mathrm{dl}$, and a reticulocyte count of $4.7 \%$ with normal white blood cell and platelet counts.

\section{Association between autoimmune hepatitis and Evans syndrome}

To our knowledge, the case report summarized above was the first case report of a pre-school aged child with a combination of AIH and ES. Tokgoz et al reported a 12-yearold female with ES, AIH and nephrotic syndrome in addition to lymphopenia, leukopenia, low IgA, IgG and IgM levels, CD3, CD4, CD8 along with low TCR alpha/beta expression, leading to a diagnosis of CD3 $\gamma$ (gamma) deficiency ${ }^{9}$.

There are other case reports of an association of AIH and ES in adults. Korkmaz et al reported a 53-year-old female with transaminitis, anemia and thrombocytopenia who was ultimately diagnosed with AIH-primary biliary cirrhosis overlap syndrome concomitant with $\mathrm{ES}^{10}$. Another case report described a 59-year-old female with $\mathrm{AIH}$ and Hashimoto's thyroiditis in association with Hodgkin lymphoma who developed ES one year later ${ }^{11}$. Another case reported ${ }^{12}$ was a 47 -year-old female with autoimmune myelofibrosis associated with AIH and concomitant ES who responded to treatment with prednisolone and rituximab similar to our case report ${ }^{1}$.

\section{Treatment of AIH and ES}

Patients with ES are difficult to manage. Although ES may initially respond to corticosteroids, it is a chronic condition with periods of exacerbation and remission. Due to side effects of chronic corticosteroid therapy, nonsteroidal therapies are often employed. The first line of treatment of ES is IVIg or corticosteroids. The secondline immunosuppressive therapies include rituximab, azathioprine, cyclosporine and mycophenolate mofetil. Other intravenous agents that have been reported as treatments for ES in adults include romiplostin and eltrombopag ${ }^{13}$. However, there is limited evidence on the use of these agents for treatment of ES and AIH in children. The efficacy of rituximab for treatment of ES in adults has been reported in a number of cases ${ }^{14-16}$. The efficacy of rituximab for children has been described in a number of hematologic conditions including treatment of AIHA, ITP, factors VIII and IX inhibitors in patients with hemophilia, post-transplant lymphoproliferative disease, Burkitt's lymphoma and others. Bader-Meunier $B$ et al reported the efficacy of rituximab along with prednisone in 14 children and in combination with other immunosuppressive drugs in 3 other children with $\mathrm{ES}^{17}$. One systemic review showed $11(67 \%)$ of $17 \mathrm{ES}$ in children achieved response, and 9 (52.9\%) achieved a complete response to rituximab ${ }^{18}$. 
Lastly, long-term treatment of pediatric AIH is usually required, with only $20 \%$ of $\mathrm{AIH}$ type 1 patients able to discontinue therapy successfully ${ }^{2}$. We reported a 2-yearold girl with AIH and ES who responded well to rituximab weekly for 4 weeks, following a short-term response to IVIg, corticosteroids, and azathioprine ${ }^{1}$. Currently, there are no randomized controlled trials to determine the most effective treatment for concomitant AIH and ES in children.

\section{Summary}

To our knowledge, this is the first report of a pre-school age child with concurrent AIH and ES. We conclude that since ES may evolve over a period of time, observation for associated autoimmune conditions should be considered in these patients. Further study is needed in order to better understand the optimum management of ES and AIH in children and adults.

\section{References}

1. Jarasvaraparn C, Imran H, Siddiqui A, et al. Association of autoimmune hepatitis type 1 in a child with Evans syndrome. World J Hepatol. 2017 Aug 18; 9(23): 1008-1012.

2. Mieli-Vergani G, Heller S, Jara P, et al. Autoimmune hepatitis. J Pediatr Gastroenterol Nutr. 2009 Aug; 49(2): 158-64.

3. Mieli-Vergani G, Vergani D. Autoimmune paediatric liver disease. World J Gastroenterol. 2008 Jun 7; 14(21): 3360-7.

4. Aladjidi N, Fernandes H, Leblanc T, et al. Evans Syndrome in Children: Long-Term Outcome in a Prospective French National Observational Cohort. Front Pediatr. 2015 Sep 29; 3: 79.

5. Norton A, Roberts I. Management of Evans syndrome. Br J Haematol. 2006; 132(2): 125-37. doi:10.1111/j.1365-2141.2005.05809.

6. Savașan S, Warrier I, Buck S, et al. Increased lymphocyte Fas expression and high incidence of common variable immunodeficiency disorder in childhood Evans' syndrome. Clin Immunol. 2007 Dec; 125(3): 224-9.

7. Seif AE, Manno CS, Sheen C, et al. Identifying autoimmune lymphoproliferative syndrome in children with Evans syndrome: a multi-institutional study. Blood. 2010 Mar 18; 115(11): 2142-5.
8. Stepensky P, Rensing-Ehl A, Gather R, et al. Early-onset Evans syndrome, immunodeficiency, and premature immunosenescence associated with tripeptidyl-peptidase II deficiency. Blood. 2015 Jan $29 ; 125(5)$ : 753-61.

9. Tokgoz H, Caliskan U, Keles S, et al. Variable presentation of primary immune deficiency: two cases with CD3 gamma deficiency presenting with only autoimmunity. Pediatr Allergy Immunol. 2013 May; 24(3): 257-62.

10. Korkmaz H, Bugdaci MS, Temel T et al. Autoimmune hepatitisprimary biliary cirrhosis overlap syndrome concomitant with immune hemolytic anemia and immune thrombocytopenic purpura (Evans syndrome). Clin Res Hepatol Gastroenterol. 2013 Apr; 37(2): e45-50.

11. Saltoh T, Matsushima T, Saito Y. Hodgkin lymphoma presenting with various immunologic abnormalities, including autoimmune hepatitis, Hashimoto's thyroiditis, autoimmune hemolytic anemia, and immune thrombocytopenia. Clin Lymphoma Myeloma. 2008 Feb; 8(1): 62-4.

12. Ohkawara H, Furukawa M, Ikeda K, et al. Steroid-resistant autoimmune myelofibrosis in a patient with autoimmune hepatitis ar $\beta$ Evans syndrome complicated with increased expression of TGF- in the bone marrow: a case report. Int J Hematol. 2017 June5.

13. Ruiz-Arguelles GJ, Ruiz-Delgado GJ, Velazquez-Sanchez-de-Cima S, et al. Simultaneous romiplostin, eltrombopag, and prednisone were successful in severe thrombocytopenia of Evans syndrome refractory to hydrocortisone, splenectomy, intravenous IgG and rituximab. Hematology. 2013 May; 18 (3): 175-7.

14. Koulova L, Alexandrescu D, Dutcher JP, et al. Rituximab for the treatment of refractory idiopathic thrombocytopenic purpura (ITP) and thrombotic thrombocytopenic purpura (TTP): report of three cases. Am J Hematol. 2005 Jan; 78(1): 49-54.

15. Ruckert A, Glimm H, Lubbert M, et al. Successful treatment of lifethreatening Evans syndrome due to anti-phospholipid antibody syndrome by rituximab-based regimen: a case with long-term followup. Lupus 2008; 17: 757-60.

16. Carey EJ, Somaratne K, Rakela J. Successful rituximab therapy in refractory autoimmune hepatitis and Evans syndrome. Rev Med Chil. 2011 Nov; 139(11): 1484-7.

17. Bader-Meunier B, Aladjidi N, Bellmann F, et al. Rituximab therapy for childhood Evans Syndrome. Haematologica. 2007; 92: 1691-1694.

18. Liang Y, Zhang L, Gao J, et al. Rituximab for children with immune thrombocytopenia: a systemic review. PloS One. 2012; 7: e36698. 